\title{
Correction to: Synthetic antimicrobial agents inhibit aflatoxin production
}

\author{
Jing $\mathrm{Li}^{1} \cdot$ Q Qing-Qing Zhi ${ }^{1} \cdot$ Jie Zhang ${ }^{1} \cdot$ Xiao-Yu Yuan ${ }^{1} \cdot$ Li-Hong Jia $^{2} \cdot$ Yu-Lin Wan ${ }^{1} \cdot$ Qiu-Yun Liu ${ }^{1} \cdot$ Jian-Rong Shi ${ }^{3}$. \\ Zhu-Mei He ${ }^{1}$
}

Published online: 26 April 2021

(C) Sociedade Brasileira de Microbiologia 2021

\section{Correction to: Brazilian Journal of Microbiology https://doi.org/10.1007/s42770-021-00423-4}

In the original publication of the article, the funding information was incomplete. This work was also supported by grants from Guangzhou Science and Technology Program (201804010328) and Science and Technology Transformation Program of Sun Yat-sen University of China (33000-18843234).

Publisher's note Springer Nature remains neutral with regard to jurisdictional claims in published maps and institutional affiliations.

The online version of the original article can be found at https://doi.org/ 10.1007/s42770-021-00423-4

Qiu-Yun Liu

lsslqy@mail.sysu.edu.cn

$\triangle \quad$ Jian-Rong Shi

jianrong63@126.com

$\bowtie$ Zhu-Mei He

lsshezm@mail.sysu.edu.cn

1 The Guangdong Provincial Key Laboratory for Aquatic Economic Animals, School of Life Sciences, Sun Yat-sen University, Guangzhou 510275, China

2 The Guangdong Provincial Key Laboratory for Biotechnology Drug Candidates, School of Bioscience and Biopharmaceutics, Guangdong Pharmaceutical University, Guangzhou 510006, China

3 Jiangsu Key Laboratory for Food Quality and Safety-State Key Laboratory Cultivation Base, Ministry of Science and Technology, Nanjing 210000, China 